\title{
The Simple Dependency Ranking System - A novel method for defence capability prioritisation
}

\author{
D.B. Lowe $^{\text {a }}$, A.L. Hadley ${ }^{a}$ and T. Pitinanondha ${ }^{\text {a }}$ \\ ${ }^{a}$ Defence Science and Technology Organisation, Canberra ACT 2600 \\ Email: donald.lowe@dsto.defence.gov.au
}

\begin{abstract}
The prioritisation of capabilities is an important activity in Defence, as decision-makers grapple with shifting factors such as costs, schedules and tasking in the face of a finite budget. While a number of methods for prioritisation exist, they usually require large amounts of input data, often in the form of judgments, and thus can be computationally and resource intensive, particularly when accounting for multiple criteria, different stakeholder perspectives and interdependent issues. A novel approach has been developed, namely the Simple Dependency Ranking System (SDRS), which provides an alternative method of arriving at a prioritised list that also takes into consideration the most important dependencies between capabilities.
\end{abstract}

The methodology of the SDRS was specifically designed to be simple and transparent. The approach essentially consists of creating two ranked lists: one of individual elements and one of pairs of elements (that correspond to element dependencies). By making assumptions with respect to the relative scoring of the ranked elements, both within each list and across each list, a final score for each element can be determined. The result is a priority ranked list that has taken the most important element linkages into account, according to the stakeholders. While initially originating from social choice theory, the SDRS is shown to be related to established methods in requirements prioritisation and network analysis.

By virtue of its design principles, the method has additional advantages: it is relatively quick to perform, it requires minimal data in its operation, it has the potential to be scalable, and it can be performed by individuals or groups. In addition, the explicit articulation of element dependencies permits the generation of a partial systems diagram that should reflect the core connected clusters of the system. As it is not comprehensive, it is unlikely to be the only method used in managing a portfolio of projects such as the DCP. However, it may be useful as a quickly repeatable approach to identifying areas of similarity and difference and a framing technique for group discussion and decision-making.

As well as introducing the method, this paper also reports on an initial experiment devised to explore and test the approach. Twelve volunteers took on roles as Defence domain stakeholders (Air, Maritime, Land and Joint) and prioritised a set of thirty-two Defence capabilities (eight from each domain). Two questions were of specific interest:

- Does the process of identifying pairs improve the consistency of the final rankings amongst players?

- Is it adequate to simply average individual responses, rather than attempt to achieve a group decision?

The results of this limited experiment show some positive evidence for both questions i.e. some convergence towards an "agreed list" and the high similarity of averaged individual results with the team decision. While only preliminary, the initial results are encouraging and indicate that the method deserves further investigation and potential refinement.

Keywords: Keywords: Prioritisation, defence capabilities, systems, dependencies 


\section{INTRODUCTION}

The Defence Capability Plan or DCP (Commonwealth of Australia, 2012) is the list of proposed projects to develop future national defence capabilities. The prioritisation of capabilities is an important activity as decision-makers grapple with shifting factors, such as costs, schedules and tasking, in the face of a finite budget. However, decisions are often made which adversely affect other projects or programs as their interdependencies were not fully appreciated at the time. The Simple Dependency Ranking System (SDRS) has been developed to help capture some of these dependencies when considering the relative priority of capabilities and the impacts of any changes to the DCP.

While many methods for prioritising projects exist, they often must be implemented at the enterprise level to be successful because of the data and resource requirements needed to implement and maintain such systems. For example, Multiple Criteria Decision Making (MCDM) methods have been used in the past, such as weighted sum models (WSM) and the Analytical Hierarchy Process (AHP), which can require numerous judgements (Nguyen, 2003). Typically for WSM with $n$ alternatives, with $m$ weighted criteria, each decisionmaker needs to make $m(n+1)$ judgements in order to arrive at a ranked list. For the same problem, a standard AHP requires at least $[n(n-1) / 2+m(m-1) / 2]$ judgements and the number of judgements and calculations generally increases as more sophisticated versions of either of these methods are applied. In addition, these methods become significantly more complex when modified to handle interdependencies, if at all.

The SDRS has been devised as a simple and spare approach that incorporates information on the perceived paramount dependencies, requiring decision-makers to make a minimum number of judgements to arrive at a relative ranked list of items. It thus offers the potential advantages of being quick to implement, transparent to decision-makers and minimal in resource requirements. This paper describes the basis for this methodology and the results of an initial experiment that provides preliminary evidence as to its validity.

\section{BASIS OF THE SDRS APPROACH}

\subsection{The Basic Concept}

The SDRS algorithm is as follows:

1. A stakeholder is asked to create two lists out of a list of $N$ items:

a. a ranked list in order of priority of the individual $N$ items; and

b. a ranked list in order of priority of the most important $N$ pairs of items that have a dependency.

2. A score (in the form of points) is allocated to each item based on their position in each list. The points are allocated linearly in any list, with the ratio between the top and bottom items being fixed at ten-to-one.

3. The number of points for each appearance of an item in both lists is summed, to provide a final score for each and every item, from which the final ranked list of $N$ items is created.

4. If there are multiple stakeholders, the scores are averaged to arrive at the "collective view".

Put simply, a stakeholder first express their preferences as a ranked list of items, and a value is attributed to each item based upon their position (primary rankings). A stakeholder then provides information on the most important dependencies between items (captured as the pair of items at either end of the link). The value of the links is transferred to these end-point items, which ultimately permits a re-ranking of the individual items based upon the summation of all attributed value. This means each stakeholder creates two lists: an initial ranked list and a final ranked list, the latter which is a modified version of the initial list (but now incorporating dependency information).

\subsection{Basis of the method}

There are a multitude of methods with which to create a single list of items from the preferences of a group of people. The following are the methods on which the SDRS is loosely based.

Rank aggregation, the task of creating a single ranked list of items by combining the ranked preferences of multiple participants, is an old problem, perhaps first studied systematically by Borda and Condorcet in the late 18th century when they proposed different voting systems (Brams et al., 2002). Known commonly as Social Choice Theory, a variety of voting systems have been generated from these two basic approaches with different modifications and additions to the basic rules. The Borda Count method allocates integer points (in reverse order) to a candidate corresponding to the position each voter puts that candidate in their list. The points from each voter are summed for each candidate and the candidate with the highest total is declared the winner. 
Requirements Prioritisation is a key phase in any systems and software engineering design, and a variety of methods exist that are commonly used by practitioners ranging from simple ranking and pair-wise comparison methods through to complex multi-criteria decision analysis (MCDA) techniques (Hull, 2005; Aurum et al., 2005; Berander et al., 2005). Rank Order methods are perhaps most closely related to the SDRS, where ranks are automatically converted to relative weights according to a pre-defined function, being useful quick approximations to the time and resource consuming process of explicitly eliciting weights (Barron et al., 1996; Jia et al., 1997). The linear allocation of points in the SDRS is reflective of the linear function used in the Rank Sum method which has been found to be a pragmatically simple approach (Roberts et al., 2002; Alfares et al., 2008).

As the SDRS incorporates dependencies, Network Analysis methods are relevant, and there are a variety of formulae available to determine the relative importance of a node in a network. These are generally known as network centrality measures and the basic versions rely on the structure of the network. Three commonly used, conceptually distinct, measures are degree, closeness and betweenness centrality (Freeman, 1979). The SDRS is most closely related to an extension of degree centrality to weighted networks (Barrat et al., 2004). While degree centrality of a node is defined as the number links incident on a node, an equivalent perspective is that it is the sum of the link weights, where all the weights are one. This can be simply extended by allowing weights to vary from one and defining this as the strength of a node. It has been argued that this extension provides more information and a more complete description of the architecture of real-world networks than the un-weighted measures alone (Barrat et al., 2004).

The preceding discussion reveals that the SDRS is related to, and can be viewed as, a distinct variation of, each of these standard methodologies listed above. The SDRS adopts a linear preference function as in Borda Counts and Rank Sum methods (but with its own rules on distributing relative value) and allocates points to a node based upon the link weights as is done in determining degree centrality in weighted networks (but in addition to the node's individual score). Thus, while it is not necessarily a direct derivation of any of the above methods, it perhaps can be more accurately viewed as a method that combines useful elements of other approaches.

\section{THE INITIAL EXPERIMENTAL INVESTIGATION}

\subsection{Experimental Method}

An experiment was conducted to investigate the use of SDRS in prioritising Defence capabilities, and identifying the factors that could impact its application. Two questions were of specific interest:

- Does the process of identifying pairs improve the consistency of the final rankings amongst players?

- Is it adequate to simply average individual responses, rather than attempt to achieve group decision?

A half-day workshop was held in 2010 with twelve civilian Defence personnel who each adopted a domain role (Air (A), Maritime (M), Land (L) or Joint (J)) according to their most prominent experience resulting in four domain teams, each with three individuals. A set of thirty-two capabilities (eight capabilities associated with each domain labelled from 1 to 8 ) that are planned to be in existence in the future, drawn from the DCP (planned capabilities) and the Key Defence Assets Review 2008 (extant capabilities), were presented to participants to consider and prioritise. These were produced as packs of cards to provide a more physical and tactile experience when sorting and ranking. Two games were conducted:

- Individual play

- Team Play (teams being based on the domains)

For each game, participants (either individually or as a team) were asked to follows the SDRS algorithm and performed the following actions:

- The thirty-two capabilities were ranked in order of personal preference.

- Sixteen pairs of capabilities ${ }^{1}$, that (from their perspectives) have a significant dependency, were identified and ranked in order of preference. In addition, they were asked to qualify the nature of the relationship between the pairs as Operational, Physical and/or Informational.

\footnotetext{
${ }^{1}$ It can be observed that there are only sixteen ranked pairs when the proposed methodology calls for thirtytwo. Due to experimental conditions and constraints, the list was truncated. However, as the original choice of $N$ pairs is somewhat arbitrary, this change does not invalidate the following results, nor the conclusions.
} 
Participants entered the prioritised lists onto data sheets which were then entered into an MS Excel ${ }^{\mathrm{TM}}$ Spreadsheet for analysis. In addition, feedback was sought from the participants via comments sheets and a discussion session where participants were asked to identify issues and problems with the approach, as well as any possible remedies or mitigating strategies.

\subsection{The correlation tests}

Two different types of tests were performed to measure the level of similarity of two lists: the Kendall's tau $(\tau)$ correlation coefficient analysis measures the degree of correlation of the orderings between two ranked lists, and a simple comparison of the amount of intersection between the top halves (subsets) of the two ranked lists, disregarding order (which we call the degree of commonality). The latter offers a qualitatively different view which corresponds more closely to the simplest pragmatic approach to making choices from a prioritised list, i.e. the items above the line are selected (regardless of order) and those below the line are rejected. This is measured from 0 (no items common to both subsets) to 1 (all items present in both subsets).

\section{3. $\quad$ Results and Analysis}

Four data sets were collected during the experiment: (1a) individual player item rankings; (1b) individual player paired rankings; (2a) team item rankings; and (2b) team paired rankings. As an example set of data, Table 1 below shows the rankings given to individual capabilities and paired capabilities by Player 1, an Air Force representative.

Table 2 shows the same rankings when done by the three Air Force representatives (players 1, 2 and 3) as a team. Similar tables were generated by the other eleven players and three teams.

Table 1 Ranking of individual and paired capabilities (links) according to Player 1 (Air Force).

\begin{tabular}{|c|c|c|c|c|c|c|c|c|c|c|c|c|c|}
\hline \multicolumn{7}{|c|}{ Ranking of Individual Capabilities } & \multicolumn{4}{c|}{ Ranking of Paired Capabilities } \\
\hline Rank & $\begin{array}{c}\text { Indiv. } \\
\text { Cap. }\end{array}$ & Rank & $\begin{array}{c}\text { Indiv. } \\
\text { Cap. }\end{array}$ & Rank & $\begin{array}{c}\text { Indiv. } \\
\text { Cap. }\end{array}$ & Rank & $\begin{array}{c}\text { Indiv. } \\
\text { Cap. }\end{array}$ & Rank & $\begin{array}{c}\text { Paired } \\
\text { Capabilities }\end{array}$ & Rank & \multicolumn{2}{|c|}{$\begin{array}{c}\text { Paired } \\
\text { Capabilities }\end{array}$} \\
\hline 1 & $A 1$ & 9 & $L 2$ & 17 & $A 7$ & 25 & $J 6$ & 1 & $A 1$ & $A 2$ & 9 & $M 1$ & $J 1$ \\
\hline 2 & $A 2$ & 10 & $A 3$ & 18 & $L 4$ & 26 & $J 2$ & 2 & $A 1$ & $A 8$ & 10 & $M 1$ & $J 3$ \\
\hline 3 & $A 8$ & 11 & $M 2$ & 19 & $M 8$ & 27 & $L 8$ & 3 & $A 1$ & $M 1$ & 11 & $J 1$ & $M 7$ \\
\hline 4 & $M 1$ & 12 & $M 6$ & 20 & $L 6$ & 28 & $L 5$ & 4 & $A 1$ & $J 3$ & 12 & $A 1$ & $A 6$ \\
\hline 5 & $J 3$ & 13 & $M 7$ & 21 & $J 1$ & 29 & $A 4$ & 5 & $A 1$ & $L 2$ & 13 & $J 1$ & $L 4$ \\
\hline 6 & $L 7$ & 14 & $M 5$ & 22 & $L 3$ & 30 & $J 8$ & 6 & $M 1$ & $M 7$ & 14 & $J 1$ & $L 5$ \\
\hline 7 & $M 3$ & 15 & $A 5$ & 23 & $J 5$ & 31 & $J 7$ & 7 & $M 1$ & $M 6$ & 15 & $J 1$ & $L 7$ \\
\hline 8 & $M 4$ & 16 & $A 6$ & 24 & $J 4$ & 32 & $L 1$ & 8 & $A 1$ & $J 3$ & 16 & $J 3$ & $M 1$ \\
\hline
\end{tabular}

Table 2 Ranking of individual and paired capabilities (links) according to the Air Force team.

\begin{tabular}{|c|c|c|c|c|c|c|c|c|c|c|c|c|c|}
\hline \multicolumn{8}{|c|}{ Ranking of Individual Capabilities } & \multicolumn{6}{|c|}{ Ranking of Paired Capabilities } \\
\hline \multirow{2}{*}{$\frac{\text { Rank }}{1}$} & \multirow{2}{*}{$\begin{array}{c}\text { Indiv. } \\
\text { Cap. }\end{array}$} & \multirow{2}{*}{$\begin{array}{c}\text { Rank } \\
9\end{array}$} & \multirow{2}{*}{$\begin{array}{c}\text { Indiv. } \\
\text { Cap. }\end{array}$} & \multirow{2}{*}{$\frac{\text { Rank }}{17}$} & \multirow{2}{*}{$\begin{array}{l}\text { Indiv. } \\
\text { Cap. } \\
M 3\end{array}$} & \multirow{2}{*}{$\frac{\text { Rank }}{25}$} & \multirow{2}{*}{$\begin{array}{c}\text { Indiv. } \\
\text { Cap. } \\
M 8\end{array}$} & \multirow{2}{*}{$\begin{array}{c}\text { Rank } \\
1\end{array}$} & \multicolumn{2}{|c|}{$\begin{array}{c}\text { Paired } \\
\text { Capabilities }\end{array}$} & \multirow{2}{*}{$\begin{array}{c}\text { Rank } \\
9\end{array}$} & \multicolumn{2}{|c|}{$\begin{array}{c}\text { Paired } \\
\text { Capabilities }\end{array}$} \\
\hline & & & & & & & & & $A 1$ & $A 2$ & & $A 1$ & $M 2$ \\
\hline 2 & $A 2$ & 10 & $A 8$ & 18 & $L 2$ & 26 & J4 & 2 & $A 1$ & $A 8$ & 10 & $A 1$ & $A 7$ \\
\hline 3 & $A 7$ & 11 & $J 8$ & 19 & $J 2$ & 27 & M4 & 3 & $A 1$ & $M 1$ & 11 & $A 1$ & $L 2$ \\
\hline 4 & $A 3$ & 12 & M6 & 20 & J6 & 28 & L6 & 4 & $A 7$ & $A 2$ & 12 & $A 1$ & $A 6$ \\
\hline 5 & $M 1$ & 13 & M5 & 21 & $J 1$ & 29 & $J 7$ & 5 & $J 3$ & $A 7$ & 13 & $A 3$ & $M 1$ \\
\hline 6 & $M 2$ & 14 & $A 5$ & 22 & $L 7$ & 30 & $L 3$ & 6 & $A 1$ & $J 3$ & 14 & $A 4$ & $A 5$ \\
\hline 7 & $J 3$ & 15 & $A 4$ & 23 & $L 5$ & 31 & $L 8$ & 7 & $M 1$ & J3 & 15 & $A 3$ & $M 1$ \\
\hline 8 & J5 & 16 & $M 7$ & 24 & $L 4$ & 32 & $L 1$ & 8 & $M 2$ & $J 3$ & 16 & $A 3$ & $A 6$ \\
\hline
\end{tabular}




\section{Question 1: Does identifying and ranking pairs improve the list correlation amongst players?}

This question was principally examined by conducting a comparison of individual responses within each domain, resulting in twelve comparisons (three comparisons within each of the four domains). The expectation here is that they should arrive at similar lists within domains (and conversely be dissimilar across domains - this was generally found but not of immediate interest in this paper). The Kendall tau value of the primary rankings (the ranking of individual capabilities) was found to range from 0.08 to 0.42 with an average of 0.26 and a standard deviation of 0.12 . The Kendall tau value of the final rankings ranged from 0.22 to 0.45 with an average of 0.33 and a standard deviation of 0.08 . These results indicate an average improvement of the tau values of 0.07 and there was a positive improvement in $75 \%$ of all comparisons.

Measuring the degree of commonality of the top half of the two rankings (primary and final) for each comparison, offers an alternative, simple and intuitive measure of list similarity. The commonality in the top half of primary rankings for the twelve comparisons of stakeholders within their domains is an average of $62 \%$ (with a standard deviation of $9 \%$ ), indicating that there is moderate-high agreement (as might be expected). Examining the final rankings, there is now an average of $69 \%$ (and a standard deviation of $8 \%$ ) indicating an average improvement of about $7 \%$.

With twelve comparisons, each with a tau value for the primary and final lists, one can test the hypothesis that the primary list tau values and the final list tau values come from the same population. It is appropriate to apply a single-tailed paired Student t-test to these two columns, from which it is found that the probability (that the two columns come from the same population) is about 3.9\%. Applying this same test in the case of the degree of commonality, it is found that the probability is about $4.6 \%$. These results are strongly suggestive that the additional capture of the dependency information in the algorithm has improved the agreement amongst the stakeholders.

\section{Question 2: Is averaging individual results the same as reaching a collective group decision?}

This question was examined by conducting a comparison of domain team rankings with the average ranking of the three individuals of that domain. In other words it is a comparison of the team average with a single deliberately crafted team response.

The primary rankings already have a moderate to high correlation with an average tau value of 0.59 (with a standard deviation of about 0.06), which is higher than any of the comparisons of the individual players discussed above. The average Kendall tau value of the final rankings increased to 0.65 (with a standard deviation of about 0.09), showing an average improvement of about 0.06. It shows both (1) the high correlation of the team average with the team decision even before adding dependency information, and (2) again an improvement in team agreement when this information is also included.

In the case of comparing the degree of commonality, both the primary rankings and the final rankings had an average commonality of $81 \%$ (and a standard deviation of about 5\%). Firstly, this implies a high level of similarity in the lists, whether rankings are performed jointly as a team, or separately as individuals. Secondly, it shows that the pairing activity made no significant improvement in the agreement of the lists. However, this observation is mitigated by the fact that the very high initial correlation of the primary rankings makes improvement in the final rankings more difficult to achieve.

Although the current sample is too small to have statistical significance, these results do indicate that the extra effort required for a team to come to an agreed decision may perhaps be unnecessary and it may be sufficient to simply aggregate the individual responses in achieving the "collectively preferred" list.

\subsection{Network Modelling}

The elicitation and ranking of dependencies allows the construction of a rich network diagram as this process identifies and captures the important linkages between the individual items. So the SDRS not only captures the priority of nodes in the network but also the priority of the linkages. Such a network diagram is constructed from the combined judgments of the twelve participants and shown in Figure 1. The first and most notable feature is that all of the nodes are a member of this continuous network (and this is despite the reduction in the number of pairs that each person had to identify, from thirty-two to only sixteen).

The SDRS method allocates scores to individual nodes and the network links. While there are no definitive boundaries, large drops in relative value (in this case $\geq 8 \%$ ), provide a natural division. Node groupings of six and twelve, and links groupings of nine and seventeen were chosen for further examination. 


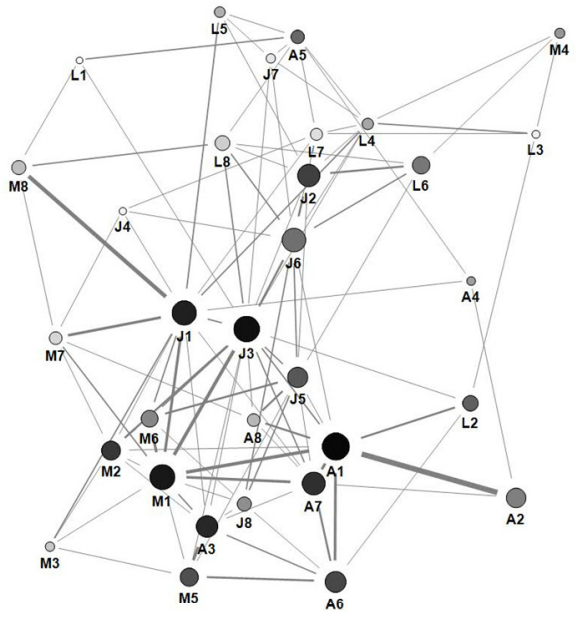

Figure 1 A network diagram of the thirty-two capabilities generated from the combined judgments of all twelve participants. There are four domains: Air, Maritime, Land and Joint (labeled A, M, L and J respectively), each with eight associated capabilities. A higher ranking is represented by the larger node size, thicker lines and darker shading (of both nodes and links).

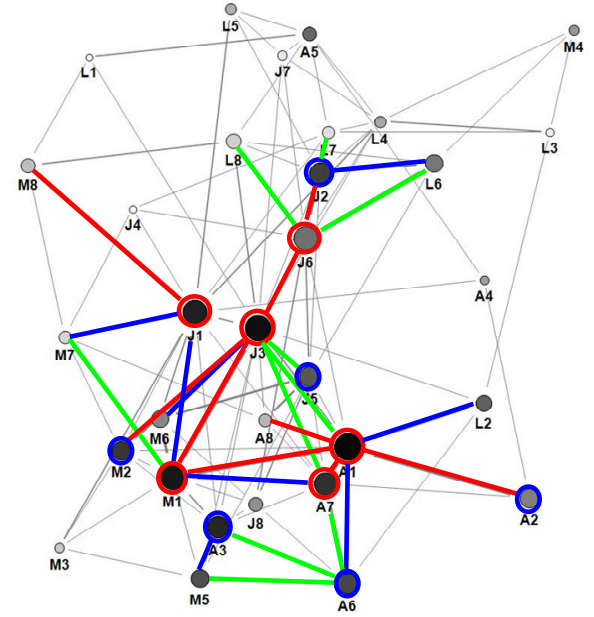

Figure 2 The sub-network created from the top 12 nodes and 27 links. The top 6 nodes and 9 links are shown in red, the next 6 nodes and 8 links are in blue and the next 10 links are in green.

Error! Reference source not found.2 shows progressive layering of most important nodes and links in the identified system, labelled by red, blue and green progressively. This shows the gradual emergence of structural features, arising just from the most important nodes and links. Principal among these are (1) the sub-network is fully connected, (2) A1, M1 and J3 figure prominently, important individually but also as nodes with multiple important links, (3) triangular loops appear, key amongst these being A1-M1-A7 as its component links are bases for other important triangular loops. This shows the benefit to be gained from the identification and ranking of pairs.

\subsection{Participant Feedback}

The feedback from the participants identified several procedural issues with the methodology:

- Context. Individuals reported some difficulties in the prioritisation task with only a broad context given.

- Visual Aids. The participants suggested that further visual aids (beyond the use of cards) would enhance the experience.

- Cognitive Load. During the experiment, the players reported significant difficulty in identifying a full thirty-two pairs so this was cut down to sixteen. The ranking of pairs was also reported to be difficult, seemingly because it felt like comparing "apples with oranges". Several possible factors have been deduced that may be at play: (1) while knowledgeable, the participants were not the true stakeholders, (2) the time available to perform the task was limited, and (3) there was limited preparation.

\section{DISCUSSION AND CONCLUSIONS}

While the results are preliminary, the analysis of the data suggests that:

- the task of identifying and ranking pairs of items generally improved agreement amongst participants; and

- the approach of canvassing individual opinion and "averaging" provides reasonably similar results to that of collective decision-making (for less effort).

Together, these provide some justification of the methodology, which has the benefits of being simple to understand, relatively quick to perform, transparent in its operation, spare in the information and minimal in the effort required of the stakeholders. These properties give it the potential to be scaled up to hundreds of items and stakeholders.

A key advantage is that the individual preferences are retained and made transparent and explicit, which has the follow-on benefit of generating a richer network diagram than would be obtained if only a collective 
group decision was sought. It also means that the extra effort of attempting to achieve a group decision is avoided, saving time and resources, and input could be gathered in a distributed and asynchronous manner. The resultant network diagram should represent the "core" sub-network, at least according to the collective wisdom of the stakeholders. Furthermore, this network diagram itself can be examined in layers: given that all the nodes and links have values, when starting from the highest valued nodes and links, the critical and significant boundaries of component sub-networks can be identified and analysed in sequence.

The technique has been shown to have connections with standard methods, but its suitability and particular applicability to the problem at hand has not yet been comprehensively tested. Furthermore, a number of starting assumptions and conditions are in operation, and their validity and influence on the results have not been fully examined as yet. For instance, the weighting and shape functions for ranking individual projects and pairs need to be explored further and robust arguments for final functional choices need to be established.

The participant feedback suggests several avenues of exploration in order to improve the robustness and efficacy of the process. In terms of context, it is possible to provide more detailed pre-reading and scenario information. However, this must be balanced against the potential anchoring of the participant's views and skewing of the outcome. The visual experience could be enhanced and streamlined by the development of a user-friendly graphical user interface, which has the added advantage of avoiding participant errors and thus addressing some aspects of the cognitive load by managing the lists as they are constructed. Other options for assisting with the mental task are the preparation of more structured information (such as the construction of a master list of dependencies) and the provision of systems that make the task more manageable (such as formal ranking methods like a binary sort).

\section{ACKNOWLEDGMENTS}

The authors wish to acknowledge the work of Emily Rawlings, Paul Norman, Victoria Sainter, Nicola Drake and Claire Reeves who assisted with some of the experimentation and analysis in this paper. Many thanks to our Fairbairn Park colleagues in the DSTO Joint and Operations Analysis Division for their critical analysis of the methodology, which has improved our understanding of its benefits and limitations.

\section{REFERENCES}

Alfares, H. K. and Duffua, S.O. (2008). Assigning Cardinal Weights in Multi-Criteria Decision Making Based on Ordinal Ranking. Journal of Multi-Criteria Decision Analysis, 15(5-6), 125-133.

Aurum, A. and Wohlin, C. (2005). Managing and Engineering Software Requirements, Springer-Verlag, Berlin Heidelberg.

Barrat, A., Barthelemy, M., Pastor-Satorras, R. and Vespignani, A. (2004). The architecture of complex weighted networks. Proceedings of the National Academy of Sciences, 101(11), 3747-3752.

Barron, F. H. and Barrett, B.E. (1996). Decision quality using ranked attribute weights. Management Science 42(11), 1515-1523.

Berander, P. and Andrews, A. (2005). Requirements prioritization. In Aurum, A. Wohlin, C. (Eds.), Engineering and Managing Software Requirements, Springer-Verlag, Heidelberg, 69-94.

Brams, S.J. and Fishburn, P.C. (2002). Voting Procedures. In Kenneth Arrow, Amartya Sen, and Kotaro Suzumura (eds.), Handbook of Social Choice and Welfare. Amsterdam: Elsevier Science, 175-236.

Commonwealth of Australia (2012). Defence Capability Plan - Public Version. Defence Publishing Service.

Freeman, L. (1979). Centrality in social networks: Conceptual clarification, Social Networks, 1, 215-239.

Hull, E., Jackson, K. and Dick, J. (2005). Requirements Engineering (2nd Edition), Springer, London Berlin Heidelberg.

Jia, J., Fischer, G.W. and Dyer, J.S. (1998). Attribute weighting methods and decision duality in the presence of response error: a simulation study. Journal of Behavioural Decision Making, 11(2), 85-105.

Nguyen, M (2003). Some Prioritisation Methods for Defence Planning. Defence Science and Technology Organisation Information Sciences Laboratory, DSTO-GD-0356.

Roberts R. and Goodwin, P. (2002). Weight approximations in multi-attribute decision models. Journal of Multi-Criteria Decision Analysis, 11(6), 291-303. 